\title{
The Feature of Solitary Small Nodular Type of Hepatic Epithelioid Hemangioendothelioma
}

\author{
Norio Kubo ${ }^{a}$ Norifumi Harimoto ${ }^{a}$ Kenichiro Araki ${ }^{a} \quad$ Kei Hagiwara ${ }^{a}$ \\ Takahiro Yamanaka ${ }^{a}$ Norihiro Ishii ${ }^{a}$ Mariko Tsukagoshi ${ }^{a}$ \\ Takamichi Igarashi $^{a} \quad$ Akira Watanabe $^{a}$ Masaya Miyazaki ${ }^{b}$ \\ Hideaki Yokooc Hiroyuki Kuwano ${ }^{d} K^{c}$ Shirabe ${ }^{a}$ \\ aDepartment of Hepatobiliary and Pancreatic Surgery, Gunma University Graduate School \\ of Medicine, Maebashi, Japan; 'bepartment of Diagnostic and Interventional Radiology, \\ Gunma University Hospital, Maebashi, Japan; 'Department of Human Pathology, Gunma \\ University Graduate School of Medicine, Maebashi, Japan; dDepartment of General \\ Surgical Science, Gunma University Graduate School of Medicine, Maebashi, Japan
}

\section{Keywords}

Hepatic epithelioid hemangioendothelioma $\cdot$ Hepatic nodule $\cdot$ Rare liver tumor

\begin{abstract}
Hepatic epithelioid hemangioendothelioma (HEHE) is a rare tumor. Preoperative diagnosis of HEHE is difficult because it does not manifest specific symptoms or tumor markers. We report a resected case of small and solitary HEHE. The patient, a 74-year-old man, had undergone surgical resection for left renal cell carcinoma 20 years ago. During follow-up, a tumor approximately $1.3 \mathrm{~cm}$ in diameter was detected by computed tomography (CT) at liver segment VIII. It showed isodensity in the arterial phase, low density in the portal venous phase, and homogeneous enhancement in the late phase on $\mathrm{CT}$ and magnetic resonance imaging (MRI). We performed hepatic resection of the right hepatic vein drainage area. A pathological diagnosis of HEHE was made. Although small and solitary HEHE is rare, an enhancement pattern in each phase on $\mathrm{CT}$ and $\mathrm{MRI}$, using contrast media, can yield clues for the diagnosis of HEHE.
\end{abstract}

(C) 2018 The Author(s)

Published by S. Karger AG, Basel 


\section{Case Reports in Gastroenterology}

Case Rep Gastroenterol 2018:12:402-410

DOI: $10.1159 / 000490524$

(c) 2018 The Author(s). Published by S. Karger AG, Basel www.karger.com/crg

Kubo et al.: The Feature of Solitary Small Nodular Type of Hepatic Epithelioid

Hemangioendothelioma

\section{Background}

Epithelioid hemangioendothelioma was first described by Weiss and Enzinger [1] in 1982 as a vascular tumor of endothelial origin that originates from soft tissue, visceral organs, bone, lung, brain, and the small intestine. Hepatic epithelioid hemangioendothelioma (HEHE) was first described as a low- to intermediate-grade malignancy [2]. It is a rare tumor with an incidence of 1 per 100,000 population [3]. HEHE commonly develops between 30 and 50 years of age, and the male-to-female ratio of incidence is 2:3 [3]. The solitary nodular form has been reported in only $13.5-18 \%$ of patients [3]. Prognosis is favorable compared to other hepatic malignancies. Long-term survival is possible with successful liver resection or liver transplantation $[3,4]$. Metastases have been reported in $27-37 \%$ of patients at presentation and occur mostly in the lungs (17\%) [3]. Laboratory analysis has shown that while liver enzymes may be moderately elevated, tumor markers are usually normal [5], making the clinical diagnosis of HEHE difficult [5]. Imaging findings of HEHE show some typical features and size-dependent patterns with contrast enhancement, on both computed tomography (CT) and magnetic resonance imaging (MRI) [5]. The small size of HEHE makes it difficult to diagnose, resulting in a misdiagnosis of cholangiocellular carcinoma or metastatic tumor. We describe here a case of small and solitary HEHE that was previously diagnosed as a metastatic tumor from renal cell carcinoma.

\section{Case Presentation}

A 74-year-old man underwent surgical resection for left renal cell carcinoma 20 years ago. During follow-ups, multidetector CT was performed at each checkup, every year. A nodule (1.3 $\mathrm{cm}$ in diameter) at liver segment VIII with a suspicion for invasion into the vein of segment VIII was detected that showed isodensity in the arterial phase, low density in the portal venous phase, and homogeneous enhancement in the late phase (Fig. 1a-c). Contrast-enhanced ultrasound with perflubutane (Sonazoid, Daiichi-Sankyo, Tokyo, Japan) showed low signal intensity at segment VIII during the Kupffer phase (Fig. 1d). 18-Fluoro-2-deoxyglucose positron emission tomography (FDG-PET) showed no abnormal uptake at segment VIII of liver or other organs. MRI showed the tumor with low intensity on T1-weighted images and high intensity on T2-weighted images (Fig. 2a, b). Dynamic MRI with Gd-EOB-DTPA-enhanced MRI showed that the tumor was not enhanced in the early phase, had low intensity relative to normal liver at 30 and $120 \mathrm{~s}$ after injection, and showed low signal intensity in the hepatobiliary phase (Fig. 2c-f). The patient tested negative for hepatitis B surface antigen and hepatitis $C$ virus antibody. Serum tumor markers like carcinoembryonic antigen, carbohydrate antigen 19-9, $\alpha$-fetoprotein, and PIVKA-2 were normal. Partial hepatectomy on the gross specimen revealed a yellowish-white mass with irregular margins (Fig. 3a). Hematoxylin-eosin staining revealed spindle- and oval to polygonal shaped cells with acidophilic cytoplasm (Fig. 3b). Tumor cells stained positive for endothelial markers (CD31, CD34, and factor VIII) (Fig. 4a-c). Tumor cells were negative for hepatocyte antigen, cytokeratin, and D2-40 (Fig. 4d-f). Based on the above data, a histological diagnosis of HEHE was made. The postoperative course was normal. The patient was discharged at 16 days post-surgery and has been relapse-free for 4 months. 


\section{Case Reports in Gastroenterology}

Case Rep Gastroenterol 2018:12:402-410

DOI: $10.1159 / 000490524$

(c) 2018 The Author(s). Published by S. Karger AG, Base www.karger.com/crg

Kubo et al.: The Feature of Solitary Small Nodular Type of Hepatic Epithelioid

Hemangioendothelioma

\section{Discussion}

HEHE is rare tumor often misdiagnosed as cholangiocellular carcinoma or a metastatic tumor. It does not have characteristic symptoms or tumor markers, making diagnosis difficult. Common symptoms include right upper quadrant pain (48.6\%), hepatomegaly $(20.4 \%)$, and weight loss (15.6\%) [3]. In our case, the patient was asymptomatic; the tumor was detected incidentally by CT, during a clinical follow-up. HEHE often presents as an advanced-stage, diffuse multifocal type and rarely as the solitary nodular type that represents an early stage [3, $4,6]$. It is important to distinguish HEHE from other hepatic tumors and to detect it before it becomes a multifocal lesion because long-term survival is possible after successful liver resection or transplantation, even in the presence of distal metastasis [7]. HEHE is difficult to diagnose using preoperative radiology techniques as seen in our study where radiological images showed a tumor, $1.3 \mathrm{~cm}$ in diameter, at liver segment VIII. A preoperative diagnosis of metastatic tumor was made. However, a pathological diagnosis subsequently revealed a small and solitary type of HEHE.

Some reports suggest the use of CT [5], MRI [8-10], and US [11] images in the preoperative diagnosis of HEHE. Zhou et al. [5] report imaging findings of HEHE that show some typical features and size-dependent patterns with contrast enhancement on both CT and MRI. Okano et al. [10] show that apparent diffusion coefficient maps may be useful in revealing the malignant potential of the tumor. Lee et al. [8] have examined the characteristic features of HEHE, divided into core and non-core groups, on MRI using Gd-EOB-DTPA. Alomari [12] first described the lollipop sign: a well-defined peripherally enhancing (or non-enhancing) lesion with an avascular core on enhanced images (the candy in the lollipop) and a histologically occluded vein, as a new cross-sectional sign of HEHE on CT and MRI. The lollipop sign rarely occurs in benign or malignant hepatic tumors; hence, it is considered a characteristic of HEHE [5]. Lesions smaller than $2.0 \mathrm{~cm}$ mostly demonstrate mild homogeneous enhancement in the arterial phase [5]. In our case, the tumor was revealed to be approximately $1.3 \mathrm{~cm}$ in diameter on CT images and showed isodensity in the arterial phase, low density in the portal venous phase, and homogeneous enhancement in the late phase. The enhanced effect of the tumor was equal to that reported by Zhou et al. [5] for a tumor when it was less than $2 \mathrm{~cm}$. In this case, the lollipop sign was unclear. Dong et al. [11] report that by analyzing hypo-enhancement in the portal venous and late phases, contrast-enhanced ultrasound can determine the malignant nature of HEHE. The role of FDG-PET in HEHE has been evaluated in previous studies. In a study of six patients, Dong et al. [13] found that 18-fluoro-2-deoxyglucose uptake in lesions of HEHE depends on tumor cellularity, not on tumor size. Dual-time-point imaging may not be useful in differentiating benign lesions from HEHE. However, the utility of FDG-PET in monitoring the effect of radiation therapy and chemotherapy has been reported $[14,15]$. These image features of HEHE are detailed in Table 1.

A diagnosis of HEHE can be made with confidence by fine needle aspiration and small biopsy with immunohistochemical staining [16]. In our case, the small tumor was located at segment VIII, between hepatic veins. Biopsy of the tumor was difficult. Therefore, the final diagnosis of HEHE required histopathological confirmation with immunohistochemical staining for endothelial markers like CD31 and CD 34, and factor VIII.

Surgical treatment options for HEHE include liver resection or transplantation; prognosis is more favorable than for other hepatic malignancies [3]. The disease-free survival rate is 
$83.3 \%$ at 1 year and $44.4 \%$ at 3 years in liver resection patients [17]. The survival rate at 1 year and 5 years, respectively, was 100 and $75 \%$ after liver resection, and 96 and $54.5 \%$ after liver transplantation [3]. Non-surgical treatment options include transcatheter arterial chemoembolization when the tumor is located only in the liver [18], and antitumor medication when the tumor has metastatic lesions with the lung or other organs. Sorafenib is a therapeutic option for unresectable HEHE. It is a tyrosine kinase inhibitor associated with this signaling pathway [19]. It is reported that, with the administration of thalidomide, the progression of diffuse metastatic HEHE is hindered successfully $[20,21]$.

HEHE is difficult to diagnose because it is a rare tumor with nonspecific symptoms and lack of tumor markers. It is important to detect the tumor early before it enters the multifocal nodule pattern stage. We found a small tumor that was treated with hepatectomy. Despite a small-sized tumor, we believe a preoperative diagnosis for HEHE to be possible by characteristic image features, as has been reported previously.

\section{Acknowledgment}

The authors thank to Yuki Saka for her secretarial assistance.

\section{Statement of Ethics}

The patient has provided permission to publish these features of his case, and his identity has been protected.

\section{Disclosure Statement}

The authors declare no conflict of interest. This study received no funding.

\section{Author Contributions}

N.K. is the first author of this manuscript and K.A. is the corresponding author. All the authors contributed to performing the surgeries, data collection, and data analysis. All authors read and approved the final manuscript. 


\section{Case Reports in Gastroenterology}

\section{References}

1 Weiss SW, Enzinger FM. Epithelioid hemangioendothelioma: a vascular tumor often mistaken for a carcinoma. Cancer. 1982 Sep;50(5):970-81.

2 Ishak KG, Sesterhenn IA, Goodman ZD, Rabin L, Stromeyer FW. Epithelioid hemangioendothelioma of the liver: a clinicopathologic and follow-up study of 32 cases. Hum Pathol. 1984 Sep;15(9):839-52.

3 Mehrabi A, Kashfi A, Fonouni H, Schemmer P, Schmied BM, Hallscheidt P, et al. Primary malignant hepatic epithelioid hemangioendothelioma: a comprehensive review of the literature with emphasis on the surgical therapy. Cancer. 2006 Nov;107(9):2108-21.

4 Baek SH, Yoon JH. Computed tomography and magnetic resonance imaging findings of a malignant hepatic epithelioid hemangioendothelioma: a rare case of solitary small nodular form. J Investig Med High Impact Case Rep. 2013 Sep;1(3):2324709613504549.

5 Zhou L, Cui MY, Xiong J, Dong Z, Luo Y, Xiao H, et al. Spectrum of appearances on CT and MRI of hepatic epithelioid hemangioendothelioma. BMC Gastroenterol. 2015 Jun;15(1):69.

6 Economopoulos N, Kelekis NL, Argentos S, Tsompanlioti C, Patapis P, Nikolaou I, et al. Bright-dark ring sign in MR imaging of hepatic epithelioid hemangioendothelioma. J Magn Reson Imaging. 2008 Apr;27(4):90812.

7 Madariaga JR, Marino IR, Karavias DD, Nalesnik MA, Doyle HR, Iwatsuki S, et al. Long-term results after liver transplantation for primary hepatic epithelioid hemangioendothelioma. Ann Surg Oncol. 1995 Nov;2(6):483-7.

8 Lee JH, Jeong WK, Kim YK, Lee WJ, Ha SY, Kim KW, et al. Magnetic resonance findings of hepatic epithelioid hemangioendothelioma: emphasis on hepatobiliary phase using Gd-EOB-DTPA. Abdom Radiol (NY). 2017 Sep;42(9):2261-71.

9 Gan LU, Chang R, Jin H, Yang LI. Typical CT and MRI signs of hepatic epithelioid hemangioendothelioma. Oncol Lett. 2016 Mar;11(3):1699-706.

10 Okano H, Nakajima H, Tochio T, Suga D, Kumazawa H, Isono Y, et al. A case of a resectable single hepatic epithelioid hemangioendothelioma with characteristic imaging by ADC map. Clin J Gastroenterol. 2015 Dec;8(6):406-13.

11 Dong Y, Wang WP, Cantisani V, D'Onofrio M, Ignee A, Mulazzani L, et al. Contrast-enhanced ultrasound of histologically proven hepatic epithelioid hemangioendothelioma. World J Gastroenterol. 2016 May;22(19):4741-9.

12 Alomari AI. The lollipop sign: a new cross-sectional sign of hepatic epithelioid hemangioendothelioma. Eur J Radiol. 2006 Sep;59(3):460-4.

13 Dong A, Dong H, Wang Y, Gong J, Lu J, Zuo C. MRI and FDG PET/CT findings of hepatic epithelioid hemangioendothelioma. Clin Nucl Med. 2013 Feb;38(2):e66-73.

14 Suga K, Kawakami Y, Hiyama A, Hori K. F-18 FDG PET/CT monitoring of radiation therapeutic effect in hepatic epithelioid hemangioendothelioma. Clin Nucl Med. 2009 Mar;34(3):199-202.

15 Shamim SA, Tripathy S, Mukherjee A, Bal C, Roy SG. 18-F-FDG PET-CT in Monitoring of chemotherapeutic effect in a case of metastatic hepatic epithelioid hemangioendothelioma. Indian J Nucl Med. 2017 JulSep;32(3):237-8.

16 Campione S, Cozzolino I, Mainenti P, D’Alessandro V, Vetrani A, D’Armiento M. Hepatic epithelioid hemangioendothelioma: pitfalls in the diagnosis on fine needle cytology and "small biopsy" and review of the literature. Pathol Res Pract. 2015 Sep;211(9):702-5.

17 Jung DH, Hwang S, Hong SM, Kim KH, Lee YJ, Ahn CS, et al. Clinicopathological features and prognosis of hepatic epithelioid hemangioendothelioma after liver resection and transplantation. Ann Transplant. 2016 Dec;21:784-90.

18 Cardinal J, de Vera ME, Marsh JW, Steel JL, Geller DA, Fontes P, et al. Treatment of hepatic epithelioid hemangioendothelioma: a single-institution experience with 25 cases. Arch Surg. 2009 Nov;144(11):1035-9.

19 Chevreau C, Le Cesne A, Ray-Coquard I, Italiano A, Cioffi A, Isambert N, et al. Sorafenib in patients with progressive epithelioid hemangioendothelioma: a phase 2 study by the French Sarcoma Group (GSF/GETO). Cancer. 2013 Jul;119(14):2639-44.

20 Mascarenhas RC, Sanghvi AN, Friedlander L, Geyer SJ, Beasley HS, Van Thiel DH. Thalidomide inhibits the growth and progression of hepatic epithelioid hemangioendothelioma. Oncology. 2004;67(5-6):471-5.

21 Salech F, Valderrama S, Nervi B, Rodriguez JC, Oksenberg D, Koch A, et al. Thalidomide for the treatment of metastatic hepatic epithelioid hemangioendothelioma: a case report with a long term follow-up. Ann Hepatol. 2011 Jan-Mar;10(1):99-102. 


\section{Case Reports in Gastroenterology}

\begin{tabular}{l|l}
\hline Case Rep Gastroenterol 2018;12:402-410 \\
\hline DOI: 10.1159/000490524 & $\begin{array}{l}\text { @ 2018 The Author(s). Published by S. Karger AG, Basel } \\
\text { www.karger.com/crg }\end{array}$ \\
\hline
\end{tabular}

Kubo et al.: The Feature of Solitary Small Nodular Type of Hepatic Epithelioid Hemangioendothelioma
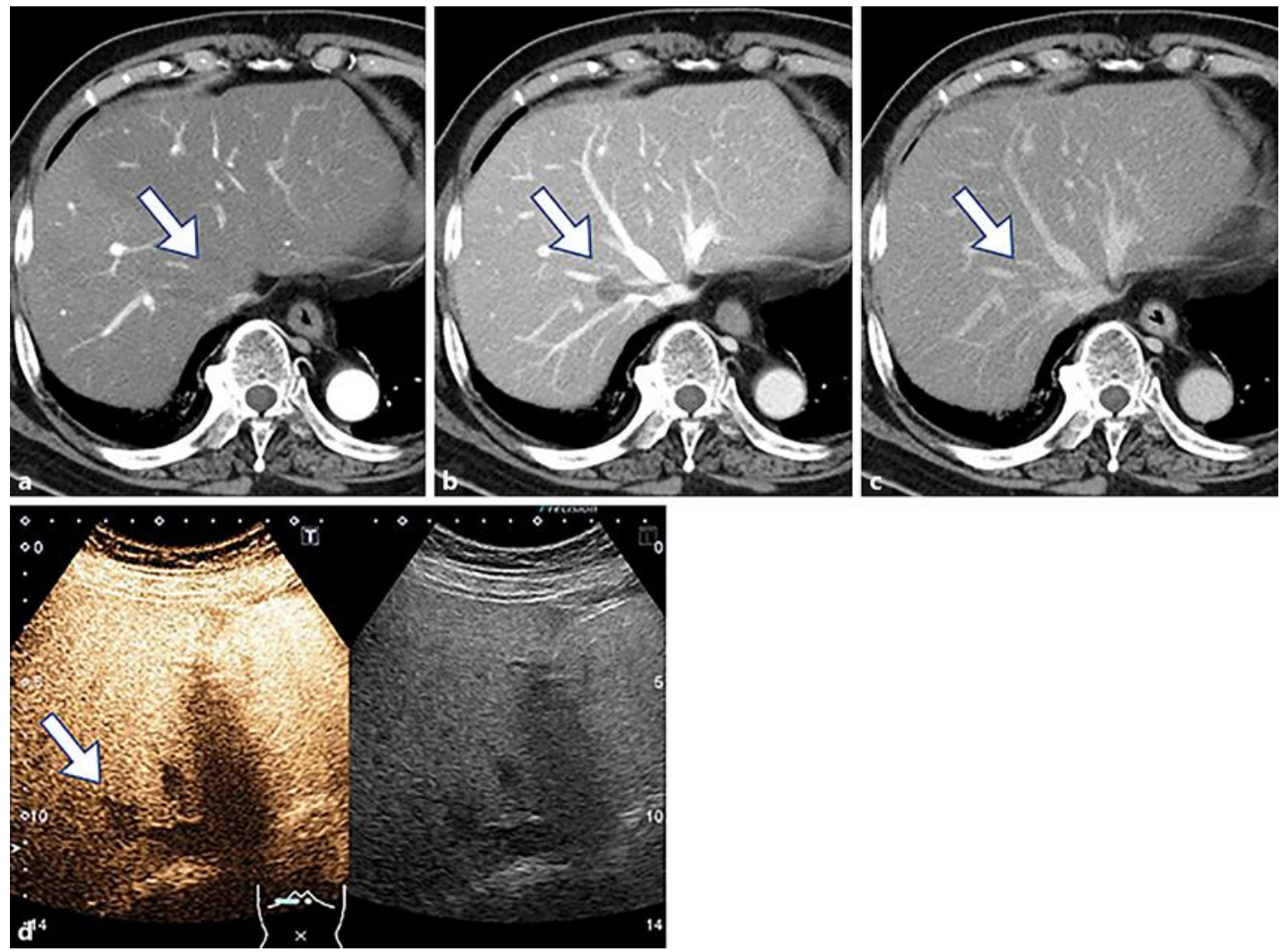

Fig. 1. Findings of dynamic computed tomography and enhanced ultrasonography. The tumor shows isodensity in the arterial phase (a), low density in the portal venous phase (b), and homogeneous enhancement in the late phase (c). Contrast-enhanced ultrasound shows low signal intensity at segment VIII during the Kupffer phase (d). 


\section{Case Reports in Gastroenterology}

Case Rep Gastroenterol 2018;12:402-410
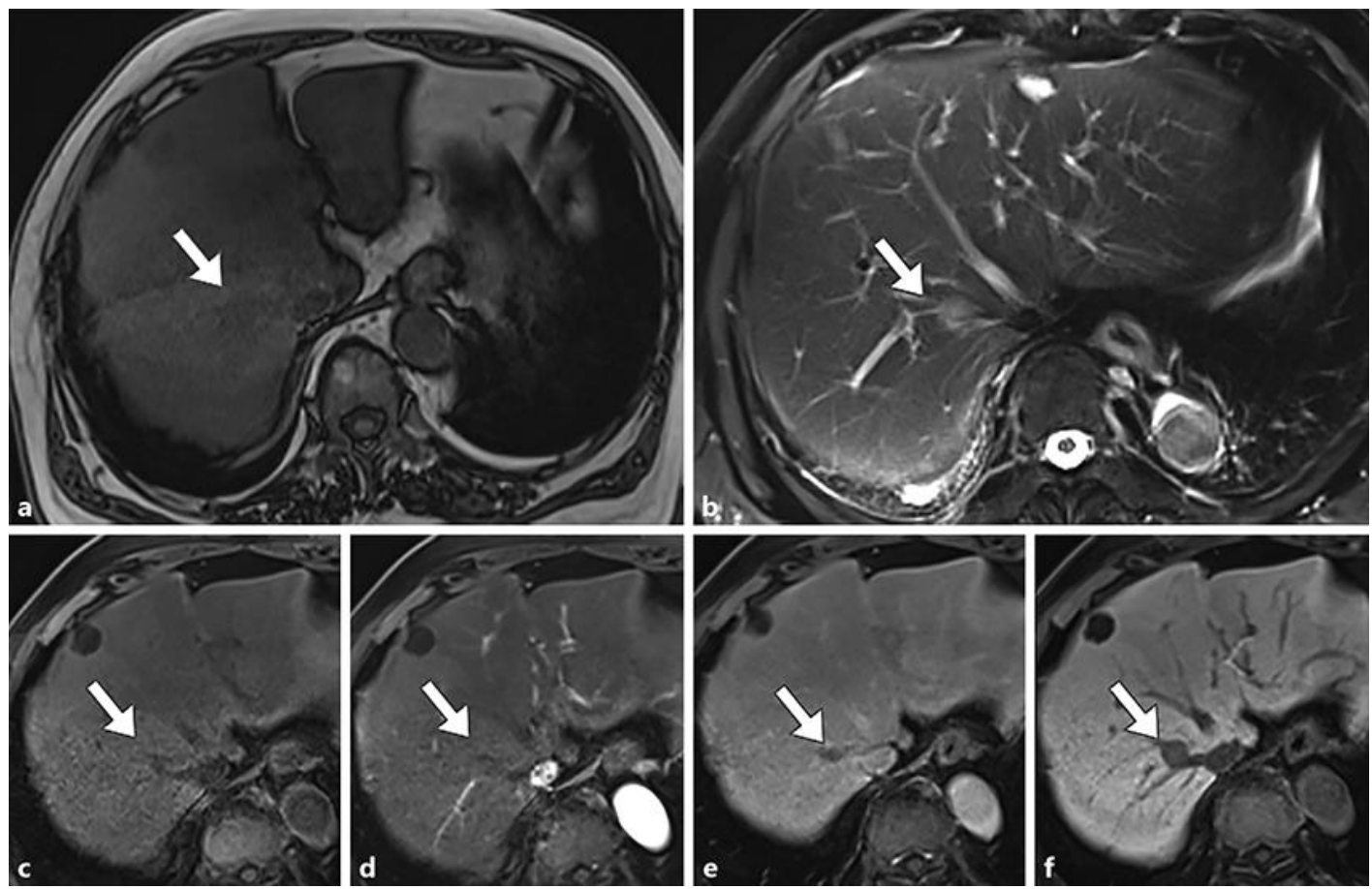

Fig. 2. Findings of magnetic resonance imaging. Magnetic resonance imaging shows the tumor with low intensity on the T1-weighted image (a) and high intensity on the T2-weighted image (b). Dynamic magnetic resonance imaging with Gd-EOB-DTPA-enhanced magnetic resonance imaging shows the tumor is not enhanced in the early phase (c), has low intensity relative to the normal liver at $30 \mathrm{~s}$ (d) and $120 \mathrm{~s}$ (e) after injection, and shows low signal intensity in the hepatobiliary phase (f). 


\section{Case Reports in Gastroenterology}

Case Rep Gastroenterol 2018;12:402-410
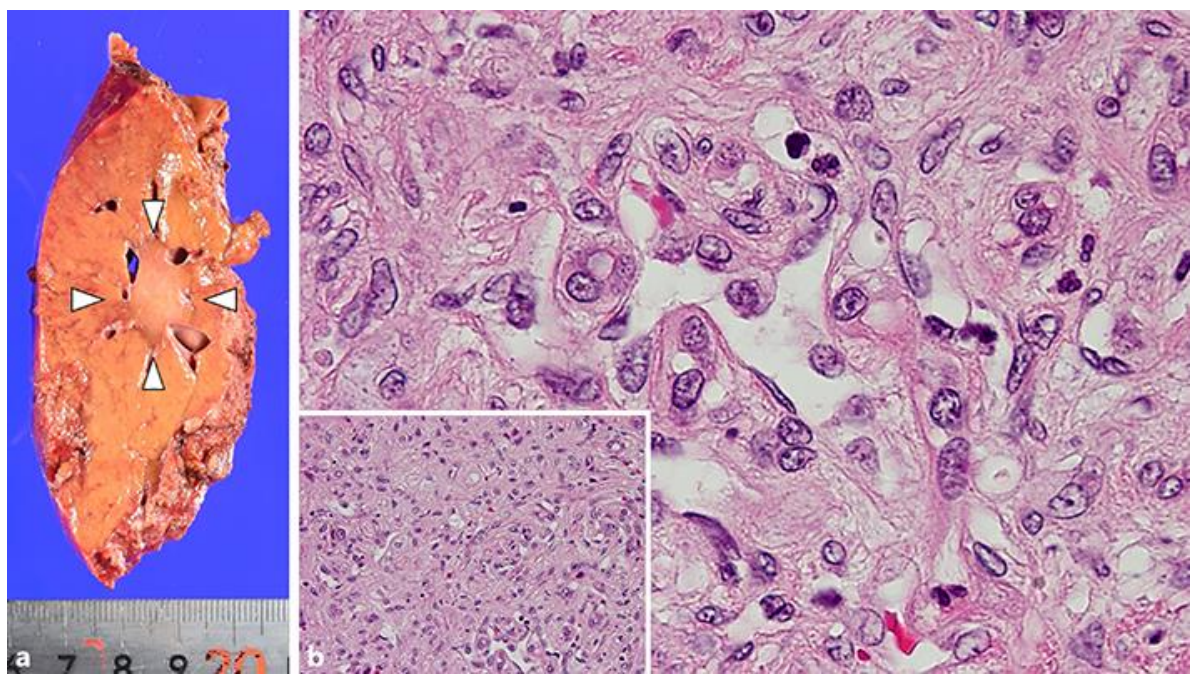

Fig. 3. Macroscopic and histopathological findings of the tumor. The cut surface of the liver explant shows a white-colored tumor, $1.7 \times 1.4 \mathrm{~cm}$ in diameter (arrowheads) (a). Hematoxylin-eosin staining shows spindle- and oval to polygonal shaped cells with acidophilic cytoplasm (b). Original magnification $\times 200$.

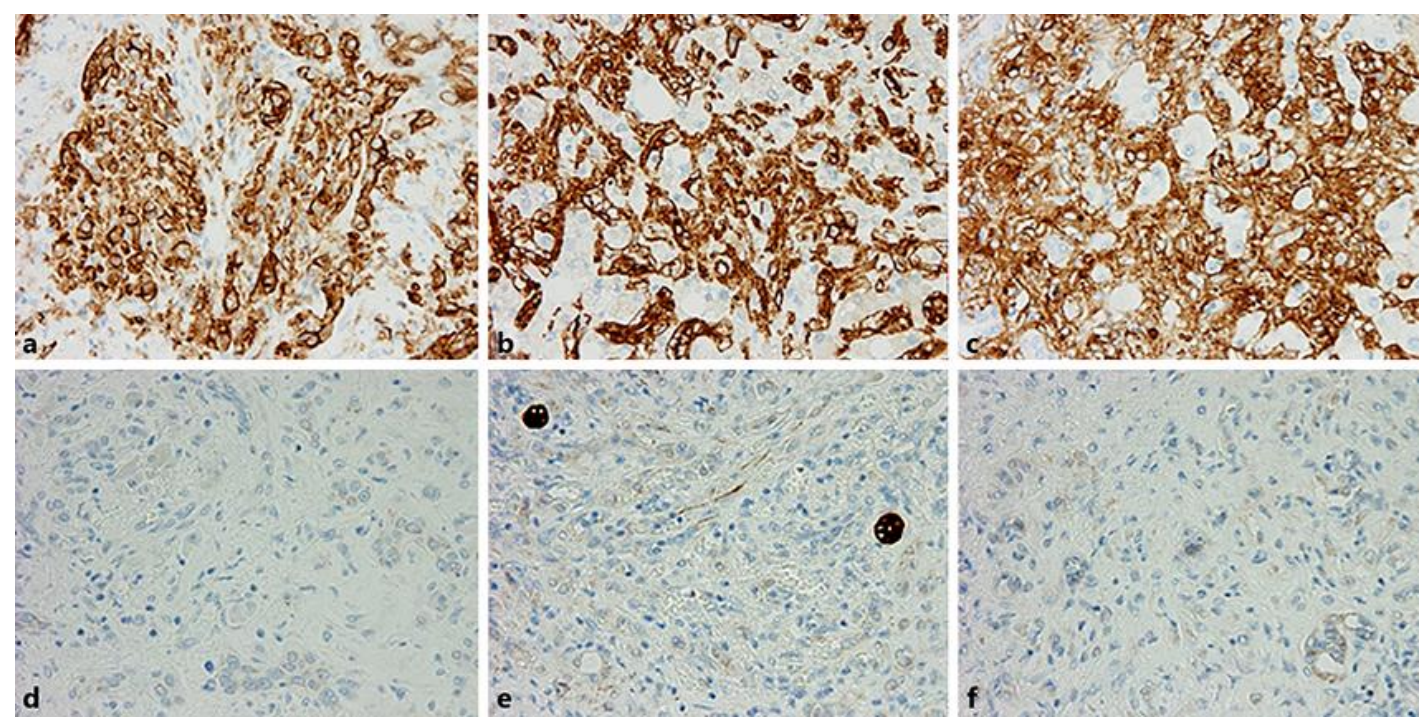

Fig. 4. Immunohistochemical staining of the tumor. Tumor cells are positive for endothelial markers CD31 (a) and CD34 (b), and factor VIII (c). Tumor cells are negative for hepatocyte antigen (d), cytokeratin (e), and D2-40 (f). Original magnification $\times 200$. 
Gastroenterology

DOI: 10.1159/000490524

\section{(C) 2018} www.karger.com/cr

Kubo et al:: The Feature of Solitary Small Nodular Type of Hepatic Epithelioid

Hemangioendothelioma

Table 1. The features of HEHE images

\begin{tabular}{|c|c|c|c|c|}
\hline Modality & Author & Pattern & Classification & Features \\
\hline \multirow[t]{3}{*}{$\mathrm{CT}$} & \multirow[t]{3}{*}{ Zhou et al. [5] } & \multirow{3}{*}{$\begin{array}{l}\text { size-dependent } \\
\text { images change }\end{array}$} & $<2.0 \mathrm{~cm}$ & mild homogeneous enhancement \\
\hline & & & $2.0-3.0 \mathrm{~cm}$ & $\begin{array}{l}\text { ring-like enhancement and heterogeneous delayed } \\
\text { enhancement }\end{array}$ \\
\hline & & & $>3.0 \mathrm{~cm}$ & heterogeneous delayed enhancement \\
\hline \multirow[t]{2}{*}{ MRI } & \multirow[t]{2}{*}{ Lee et al. [8] } & $\begin{array}{l}\text { ring-like } \\
\text { enhancement }\end{array}$ & T1WI, T2WI & $\begin{array}{l}\text { hypo- and hyperintensity of the rim compared to the signal } \\
\text { intensity at the center of the mass }\end{array}$ \\
\hline & & core pattern & hepatobiliary phase & seed-like and distinct center of low signal intensity \\
\hline $\mathrm{CT} / \mathrm{MRI}$ & Alomari [12] & lollipop sign & & $\begin{array}{l}\text { a hepatic or portal vein terminating at or just within the } \\
\text { periphery of some of the liver lesions }\end{array}$ \\
\hline \multirow[t]{2}{*}{ US } & \multirow[t]{2}{*}{ Dong et al. [11] } & & in arterial phase & rim-like or heterogeneous hyperenhancement \\
\hline & & & $\begin{array}{l}\text { in portal venous and late } \\
\text { phase }\end{array}$ & hypoenhancement \\
\hline PET & Dong et al. [13] & & & $\begin{array}{l}\text { FDG uptake of HEHE may be related to tumor cellularity } \\
\text { mean maximum standardized uptake value of all lesions was } \\
3.6 \pm 1.1\end{array}$ \\
\hline
\end{tabular}

